\title{
Study on the total reactive compensation method of offshore wind farm
}

\author{
Feng Peilei ${ }^{1}$, Wu Hesong ${ }^{* 2}$, Zhang Mingsheng ${ }^{3}$, Wan Wenkui ${ }^{3}$ \\ ${ }^{1}$ School of Electrical and Information TechnologyYunnan Minzu University Kunming, China \\ ${ }^{2}$ Diqing Grid Bureau, Yunnan Grid Corporation Limited, Diqing StateDiqing, China \\ ${ }^{3}$ Kunming University of Science and Technology Oxbridge College Kunming, China sci7663571@163.com
}

\begin{abstract}
Wind power generation is one of the most mature and most developed conditions for power generation in new energy generation technology.Large capacity and long distance offshore wind power is the trend of future wind power development.Wind power generation is one of the most mature and commercialized power generation methods in the field of renewable energy utilization.Due to the rich characteristics of offshore wind resources and the urgent need for offshore wind power development projects all over the world, the transmission and interconnection of large-scale offshore wind farms has become a hot topic in the development and research of wind power.This paper focuses on the analysis of the reactive power compensation methods for offshore wind farms, and provides a reference for the construction of offshore wind farms.
\end{abstract}

\section{Introduction}

With the rapid development of the global economy, the demand for energy is increasing.The fossil energy is drying up, and the global environmental pollution is becoming more and more serious. The search for new renewable and clean energy has become the most important issue in twenty-first Century.Wind energy, as a clean renewable energy, has received worldwide attention in recent years, especially in the field of wind power generation ${ }^{[1]}$. Various countries have stepped up a variety of policies to promote the development of the wind power industry.Wind farms can be divided into two types of land and sea.The development of the world's offshore wind power is mainly concentrated in Europe.The sea wind power has been developed in Europe for more than 20 years.In recent years, the basic development of land wind power resources in Europe, especially in Britain, Denmark, Germany and other countries has been completed.The development of marine wind power has been put on the agenda again, as the demand for reducing greenhouse gases and increasing the proportion of renewable energy.It is noteworthy that the European Union formulated the goal of renewable energy to account for $20 \%$ of the total demand and energy in 2020, and put forward the goal of offshore wind power installed capacity of 40 thousand MW by $2020^{[2]}$. With the rapid development of coastal wind power resources in China, the offshore wind power industry has made substantial progress. The total installed capacity of offshore wind power in China is expected to reach 15 million $\mathrm{kW}$ in 2015, and it is expected to reach 30 million $\mathrm{kW}$ in 2020 , which is equivalent to the total installed capacity of wind power in the country.

\section{Modeling of offshore wind power system}

\subsection{Wind power generation system}

According to the classification of wind power generation system, the constant speed and constant frequency refers to keep the generator's speed unchanged during the process of wind power generation, thus obtaining a constant frequency ${ }^{[3]}$.

It is generally used to use the wind energy coefficient $\mathrm{Cp}$ to show the efficiency of the wind turbine converting wind energy into mechanical energy.It is the function $\beta$ of the tip speed ratio $\lambda$ and the pitch angle of the blade paddle

It is known from the wind energy utilization factor of a typical wind turbine that when the pitch angle is fixed, the $\beta$ wind energy utilization coefficient CP can only reach the maximum value $\mathrm{C}_{\mathrm{P}} \max$.

Under the change of wind speed, $\mathrm{Cp}$ obviously cannot be kept at the best value, which leads to low utilization of wind energy. 


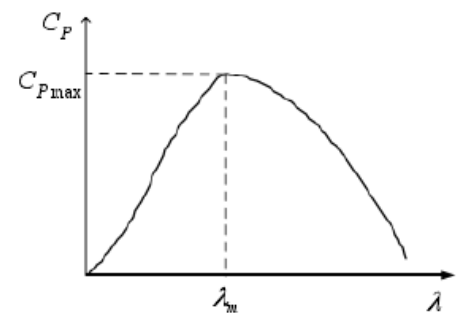

Figure 1. Typical wind turbine $\mathrm{Cp}-\lambda$ curve

\subsection{The composition of the offshore wind power system}

At present, the offshore wind farms have been built up and are being built in the world.

The composition of the marine wind power system can be obtained

As shown in the following figure:

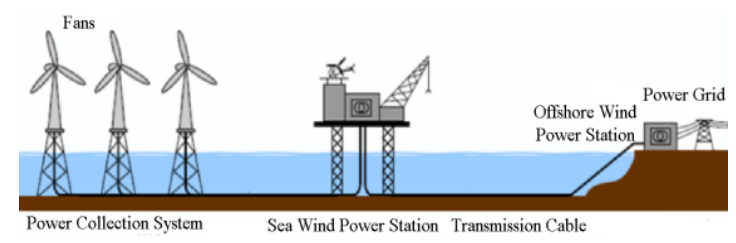

Figure 2. A schematic diagram of the composition of the offshore wind power system

From the above diagram, we can see that the main components of offshore wind power system are as follows: wind power stand-alone system, offshore power station, distance transmission and shore power plant.

The various components of the offshore wind power system are very important.

No matter which problems occur, the system can not operate normally or even destroy the stability of the power grid.

The prototype of offshore wind turbines is adapted from land based wind turbines. In addition to the great changes in the usage environment of wind turbines, the electrical principles of wind power generation are the same.

The modeling of the offshore wind power system can be referred to the land wind power system.The composition of the offshore wind power system includes the wind power generation single machine system, the power collection system, the sea boost station, the submarine cable and the upland boost station.

According to the composition module of the offshore wind power system, the energy transfer relationship of the wind power generation is combined.

The following schematic diagrams are used to represent the modules that need to be modeled:

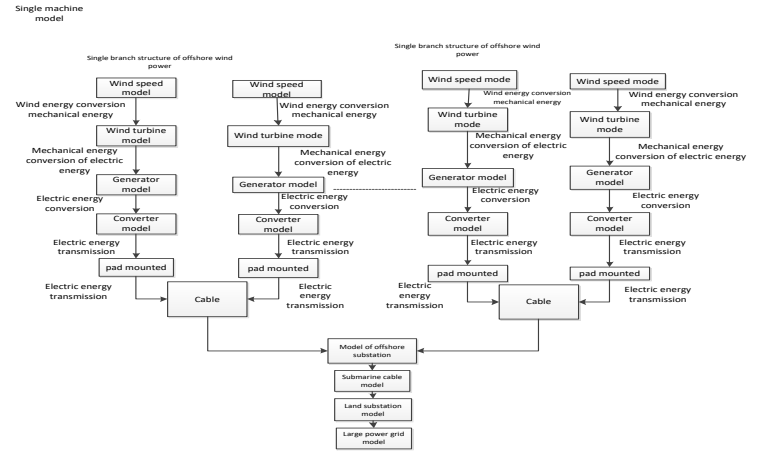

Figure 3.Block diagram of each modeling module of sea wind power system

The large size and complex structure of the large wind turbine bring difficulties to the research work of the wind power generation system.Through the establishment of the mathematical model of the wind turbine, it is an effective method to make use of computer simulation to study the wind turbine.In general, the modeling method of mechanism modeling is used to model the large variable speed variable propeller wind turbine system.

First, the sub model of each component of the wind turbine is obtained by the mechanism analysis method, and then the mathematical model of the whole unit is synthesized.

\section{The Sea Wind Model}

Wind energy is a kind of energy with low energy density and poor stability ${ }^{[4]}$.

In the process of wind movement, there are both kinetic energy changes and potential energy changes. The continuous change of wind speed is random in a certain time and space.According to the total and long term statistical results, the change of wind speed still has a certain distribution law.In order to simulate the variation of wind speed over time on the wind wheel, it is very important to establish a reliable wind speed model ${ }^{[5]}$.

1) Basic wind

The basic wind exists in the normal operation of the wind turbine.It determines the input power rating of the wind turbine to the system, which basically reflects the change of wind power average wind speed.It can be approximately determined by the Weibull distribution parameters obtained from wind power plant wind power measurement.

$$
\bar{V}=A \Gamma\left[1+\frac{1}{K}\right]
$$

In the formula: $\boldsymbol{V}$ is the basic wind speed s; $\boldsymbol{A}$ is the scale parameter of Weibull distribution; $\boldsymbol{K}$ is the shape parameter of Weibull distribution; $\Gamma(*)$ is a gamma function.

For the basic wind speed model, it is not necessary to consider the distribution of the actual wind speed in the laboratory simulation. Only considering the average wind speed acting on the wind turbine, the basic wind model specifies a specific mean wind speed. 


\section{2)Gust of wind}

In the process of wind speed change, the characteristic of sudden change of wind speed is described, which is usually expressed in gust.A gust of wind component $\boldsymbol{V}_{\boldsymbol{W}}$ can be superimposed on the basic wind.

$$
V_{W G}=\left\{\begin{array}{l}
0, t \leq T_{1 G} \\
V_{\cos }, T_{1 G} \leq t \leq T_{1 G}+T_{G} \\
0, \mathrm{t} \geq T_{1 G}+T_{G}
\end{array}\right.
$$

$$
V_{\cos }=\frac{\max G}{2}\left[1-\cos 2 \pi\left(\frac{t-T_{1 G}}{T_{G}}\right)\right]
$$

$\mathrm{V}_{\mathrm{WG}}$ is gust wind speed; $\mathrm{T}_{\mathrm{G}}$ is a cycle; $\boldsymbol{T}_{\boldsymbol{I}}$ is the starting time of gust.max $\boldsymbol{G}$ is the Maximum gust

3) The gradient wind

The gradient characteristics of the wind speed can be simulated with the gradual wind, and a gradient wind component $\boldsymbol{V}_{W R}$, it can be superimposed on the basic wind:

$$
\begin{gathered}
V_{W R}=\left\{\begin{array}{l}
0, t<T_{1 G} \\
V_{\text {ramp }}, T_{1 R} \leq t<T_{2 R} \\
\max R, T_{2 R} \leq t<T_{2 R}+T_{R} \\
0, \mathrm{t} \geq T_{2 R}+T_{R}
\end{array}\right. \\
V_{\text {ramp }}=\max R\left[1-\frac{\mathrm{t}-T_{2 R}}{T_{1 R}-T_{2 R}}\right]
\end{gathered}
$$

In the formula: $\boldsymbol{V}_{\boldsymbol{W R}}$ is gradient wind speed;maxR is the maximum value; $\boldsymbol{T}_{\boldsymbol{I}}$ is the starting time;

$\boldsymbol{T}_{2 \boldsymbol{R}}$ is the terminating time; $\boldsymbol{T}_{\boldsymbol{R}}$ is the holding time

4) Random noise wind speed

In order to reflect the randomness of wind speed change, the wind speed simulation can be used to represent the wind velocity components of random noise. A random wind volume $V_{W N}$ can be superimposed on the basic wind

$$
V_{W N}=2 \sum_{i=1}^{N}\left[S_{V}\left(\mathrm{w}_{i}\right)\right]^{1 / 2} \cos \left(w_{i} t+\varphi_{i}\right)
$$

$$
\begin{aligned}
& \mathrm{w}_{i}=\left(\mathrm{i}-\frac{1}{2}\right) \bullet \Delta w \\
& S_{V}\left(\mathrm{w}_{i}\right)=\frac{2 K_{N} F^{2}\left|w_{i}\right|}{\pi^{2}\left[1+\left(F w_{i} / u \pi\right)^{2}\right]^{4 / 3}}
\end{aligned}
$$

In the formula: $\phi_{i}$ represents a random variable of uniform distribution.

$\boldsymbol{K}_{N}$ is the surface roughness coefficient; $\boldsymbol{F}$ represents the range of disturbance; $u$ is a relatively high average wind speed; $\boldsymbol{N}$ can take a value of $50 ; \boldsymbol{U}$ is the average velocity of the relative height.

Four kinds of wind speed components are combined to simulate the wind speed of the actual effect on the wind turbine:

$$
V_{W}=V_{W B}+V_{W G}+V_{W R}+V_{W N}
$$

$V_{W}$ represents the wind speed on the wind turbine

\section{Wind turbine model}

For a variable speed constant frequency wind power generation system, the mechanical energy absorbed by the wind turbine is directly converted into electrical energy or temporarily transformed into the kinetic energy of the blade.It is stored in a wind turbine in the form of kinetic energy and then converted to power ${ }^{[6]}$.The utilization factor of wind energy utilization directly reflects the utilization ratio of wind power system to wind energy.How to maintain the highest utilization coefficient of wind energy in the changing wind speed is one of the targets of the control of the variable speed wind power generation system ${ }^{[7]}$.The wind is acting on the blade of the wind wheel at a certain speed and angle of attack, so that the blade rotates with a rotating moment.The wind energy is converted into a mechanical distance that acts on the hub and drives the generator directly or through the transmission mechanism.

According to the air characteristics of the fan blade, the utilization coefficient of wind energy is a function of the blade tip speed ratio and the pitch angle.That is:

$$
\mathrm{Cp}=\mathrm{Cp}(\lambda, \beta)
$$

The utilization efficiency of the wind power generation system can be represented by the wind energy utilization coefficient $\mathrm{Cp}$ of the wind turbine.The coefficient of utilization of wind energy is the ratio of the mechanical energy absorbed by the wind turbine to all the wind energy that is rotated through the wind turbine.

The kinetic energy contained in the air of a rotating surface of a wind turbine is:

$$
P=\frac{1}{2} \rho A V_{\mathrm{w}}^{3}=\frac{\pi}{8} \rho D^{2} V_{\mathrm{w}}^{3}
$$

The mechanical energy that the wind turbines capture from the wind is part of the kinetic energy of the air.

The mechanical output power of the wind turbine is as follows:

$$
P_{\mathrm{r}}=\frac{1}{2} \rho A V_{\mathrm{w}}^{3} C_{p}=\frac{\pi}{8} \rho D^{2} V_{\mathrm{w}}^{3} C_{p}(\lambda, \beta)
$$

In the formula: $\boldsymbol{\rho}$ is the density of air; $\boldsymbol{A}$ represents the swept area of the wind wheel.

$\boldsymbol{D}$ represents the diameter of the wind wheel; $\boldsymbol{V}_{\boldsymbol{W}}$ is air velocity; $\boldsymbol{C p}$ is the coefficient of wind energy utilization;For a fan, the wind energy absorbed depends on the wind energy utilization coefficient $\boldsymbol{C p}$ and the wind speed $\boldsymbol{V}_{\boldsymbol{W}}$.

\section{Analysis of simulation results}

PSCAD simulation diagram of the scheduling algorithm for each equivalent fan: 


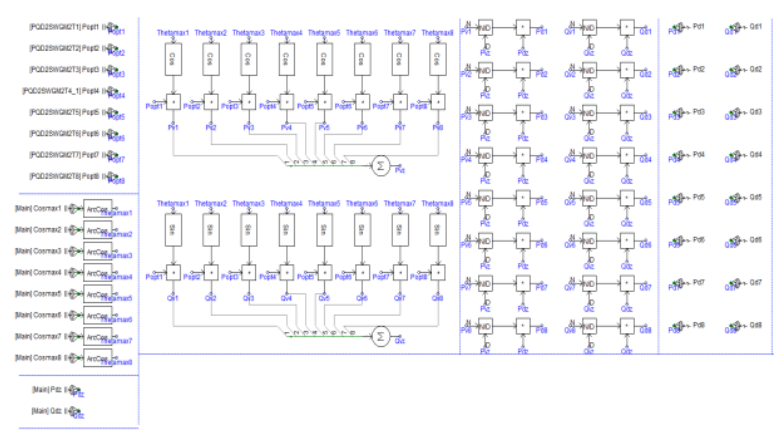

Figure 4. PSCAD simulation diagram of the equivalent fan scheduling algorithm

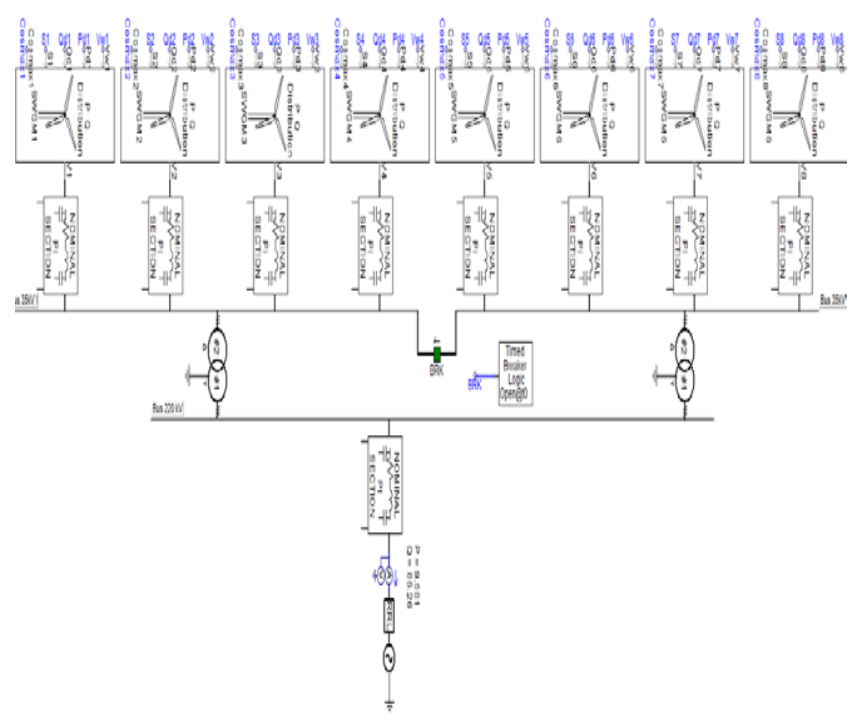

Figure 5. Main circuit diagram of PSCAD simulation for offshore wind power system

The relationship between the output voltage, current, and power of a single chain winder equivalent system is shown as follows:

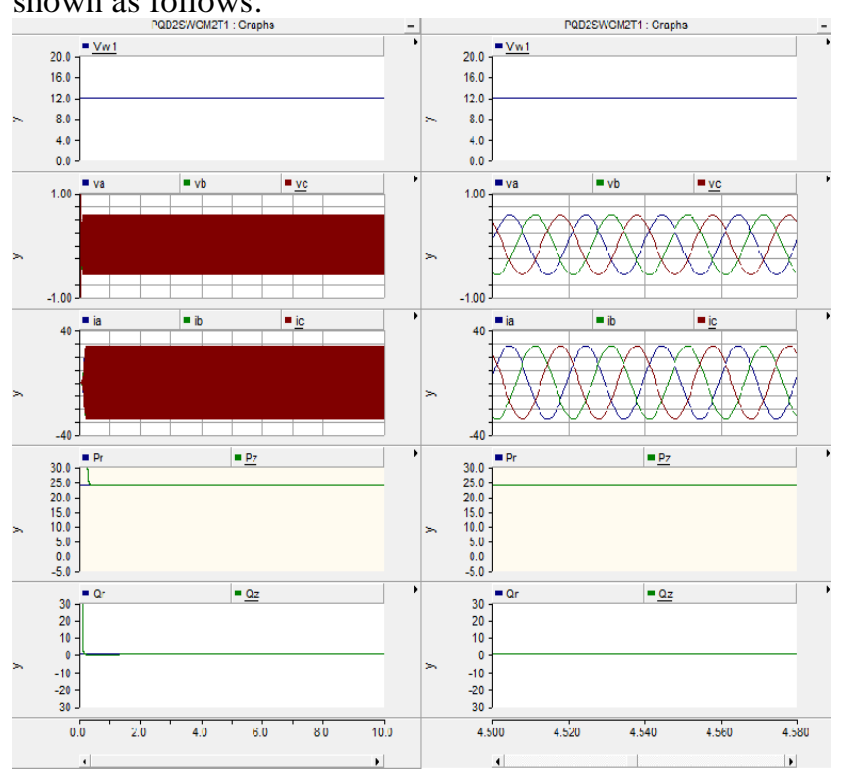

Figure 6.The waveform of the output voltage, current and power of the equivalent system of single chain winder
From the above analysis, the output voltage and current phase of the fan are equal, and the output reactive power of the equivalent Multimachine System is zero.

The output active power and reactive power of a single chain Multimachine System satisfy the system's performance with maximum active power output.

When the system is running in this state, the diagrams of the power flow voltage, current and power of the main branches are shown below. (taking branch 3 and branch 4 as examples, the other branches are similar).
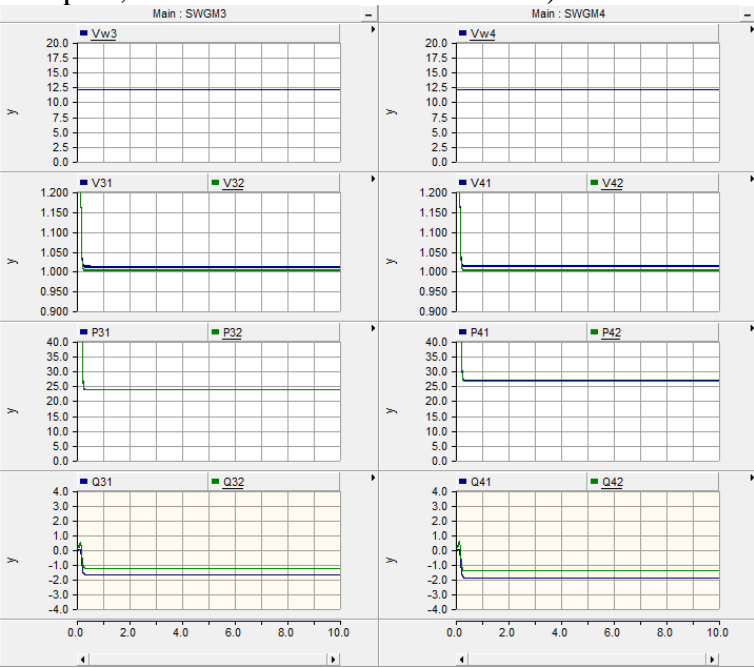

Figure 7.The electrical quantity waveform of the 3 and 4 ends of the equivalent multi machine single chain system

From Figure 7, we can see:The power and reactive power loss (including positive and negative) will be involved in the transmission of active and reactive power when the system passes through the system.From the above drawings, it can be seen that the range of the voltage is within the range of 1.0-1.05, all of which meet the requirements of the grid standard.

\section{6 summary}

New energy generation has become a hot spot of research The technology of wind power generation is becoming more and more mature, and the development of large-scale offshore wind power is of certain significance. It can be used as a distributed power supply for residential area. In this paper, a mathematical model for the development of offshore wind power is established and the wind speed is modeled.It has a certain theoretical guiding significance for the development of offshore wind power.

\section{References}

1. Wang Peng Study on dynamic coupling analysis and optimization design of three pile structures for offshore wind single column "Ocean University of China", 2011

2. Chen Xiaoming, Li Lei, Wang Hongmei, Liu Yanxing,Jiang Feng General situation and Countermeasures on the development of offshore 
wind power"Guangdong shipbuilding", 2011,30 (1): 23-28.

3. Qiu fengrong The main program "VSCF wind power generation system" machinery and equipment, 2009 (4): 69-71

4. Liu Baolan, Wenhua Li The current situation and Prospect of wind power generation in the world, energy engineering, 2000 (4): 12-14

5. Zun Pengliu Study on assessment and analysis system of "Lanzhou University of Technology" wind parameters in the control device of wind turbine, 2009

6. Rong Hua Du Research on Dynamic Characteristics Simulation and control system of wind turbines "North China Electric Power University (Hebei)", 2008

7. Qi Xiaojun, Wei Yili The wind wheel of the wind turbine modeling and simulation study of "Journal of Inner Mongolia University of Science and Technology, 2010,29 (4): 364-367 